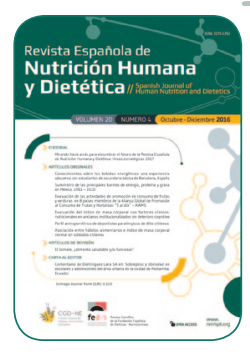

\title{
ORIGINAL
}

\section{Conocimientos sobre las bebidas energéticas: una experiencia educativa con estudiantes de secundaria básica de Barcelona, España}

\author{
Violeida Sánchez-Socarrás ${ }^{\mathrm{a}, *},{ }$ Meritxell Blanco ${ }^{\mathrm{b}}$, Carme Bosch $^{\mathrm{c}}$, Cristina Vaqué $^{\mathrm{a}}$

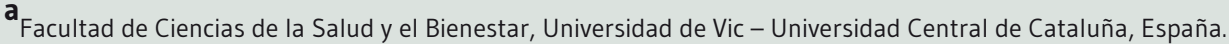 \\ ${ }^{\text {b }}$ Centro Médico Salut Solucions, Mútua Manresana, Manresa, España. \\ CFacultad de Ciencias Sociales de Manresa, Universidad de Vic - Universidad Central de Cataluña, España. \\ *vsanchez@umanresa.cat
}

Recibido el 14 de mayo de 2016; aceptado el 24 de octubre de 2016.

\section{PALABRAS CLAVE}

Educación para la

Salud;

Bebidas Energéticas;

Cafeína:

Aprendizaje.
Conocimientos sobre las bebidas energéticas: una experiencia educativa con estudiantes de secundaria básica de Barcelona, España

\section{RESUMEN}

Introducción: La popularidad de que gozan las bebidas energéticas entre los adolescentes y jóvenes, ha aumentado en los últimos años. El aumento del consumo entre los estudiantes motivó el diseño de un taller educativo en un grupo de adolescentes, con el objetivo de aumentar el nivel de conocimientos sobre las características de estos refrescos y sobre sus posibles efectos para la salud; así como de evaluar el impacto del taller respecto a estos conocimientos.

Material y Métodos: Se realizó un estudio de intervención con evaluación pre- y post- intervención en un instituto de Manresa, Barcelona. Se aplicó un cuestionario de conocimientos sobre las Bebidas Energéticas antes y después de realizar un taller educativo, en el que se emplearon técnicas de enseñanza afines a los Estilos de Aprendizaje preferentes de los participantes.

Resultados: Las técnicas de enseñanza, correspondieron a las estrategias preferentes para los estilos auditivo $(32,3 \%)$ y quinestésico $(23,7 \%)$ predominantes en el colectivo. En la evaluación previa: el 76,3\% de los adolescentes definen las Bebidas Energéticas como isotónicas para deportistas, la mayoría reconocen su elevado contenido en cafeína (95\%), el 61,3\% considera que aportan energía para el deporte, el $76,3 \%$ que pueden provocar nerviosismo y el $28 \%$ creen que no han de ser consumidas por adolescentes. En la evaluación posintervención aumentó el número de estudiantes que respondió correctamente en todas las preguntas del cuestionario.

Conclusiones: Un gran número de adolescentes tiene poco o nulo conocimiento sobre las características de las bebidas energéticas y las consecuencias de su consumo para la salud, que mejora significativamente tras la realización de un taller a través de técnicas de enseñanza participativas y cooperativas afines a las estrategias de aprendizaje preferente de los participantes. 
Knowledge about energy drinks: an educational experience in high school students from Barcelona, Spain

\section{KEYWORDS}

Health Education;

Energy Drinks;

Caffeine;

Learning.

\begin{abstract}
Introduction: Recently there has been an increase in energy drink consumption in young and adolescents, leading to caffeine abuse. This increased consumption served as motivation for creating an educational workshop in a collective of adolescents. The aim of this work was improving the level of knowledge about energy drinks and to assess the impact on the knowledge of the workshop.
\end{abstract}

Material and Methods: It was performed an intervention study with pre- and post- intervention assessment in a high school in Manresa, Barcelona. A questionnaire about energy drinks was applied before and after performing an educational workshop designed with teaching techniques, according to the preferred Learning Styles.

Results: The learning techniques used in the workshop, corresponded to the preferred strategies for aural style (32.3\%) and kinesthetic style (23.7\%). In the previous assessment: $76.3 \%$ of adolescents define Energy Drinks as isotonic drinks, the most recognize their high caffeine content (95\%); $61.3 \%$ consider that provide energy for the sport, $76.3 \%$ that can cause nervousness and $28 \%$ believe that they must not be consumed by adolescents. In the post-intervention assessment increased the number of correct answers to all questions in the questionnaire.

Conclusions: Most of the adolescents have little or no knowledge about energy drinks and about health consequences of their consumption. This knowledge increased significantly after an educational workshop with participatory and cooperatives techniques which were selected according to the preferred learning strategies of the participants.

\section{CITA}

Sánchez-Socarrás V, Blanco M, Bosch C, Vaqué C. Conocimientos sobre las bebidas energéticas: una experiencia educativa con estudiantes de secundaria básica de Barcelona, España. Rev Esp Nutr Hum Diet. 2016; 20(4): 263-72. doi: 10.14306/renhyd.20.4.228

\section{INTRODUCCIÓN}

Las bebidas energizantes o bebidas energéticas representan un conjunto de productos que desde hace algunos años se comercializan libremente en muchos países. Su irrupción en el mercado está influenciada por intensas campañas publicitarias, que las definen como bebidas refrescantes creadas para incrementar la resistencia física, proporcionar sensación de bienestar y estimular el metabolismo, capaces de suministrar un elevado nivel de energía proveniente de una combinación de ingredientes adicionados por sus posibles efectos estimulantes. Contienen una mezcla de compuestos que incluyen la cafeína, glocuronolactona y vitaminas. Algunas atribuyen sus propiedades estimulantes a aminoácidos como la taurina o a su contenido en extractos de hierbas como el ginseng o el guaraná, y en algunos casos se agregan minerales, inositol y carnitina ${ }^{1-5}$.
El reciente aumento del consumo de bebidas energéticas asociado al ocio, durante los períodos de exámenes, en relación con la práctica de actividades deportivas, así como la popularidad de que gozan entre los adolescentes y jóvenes, ha despertado la preocupación de algunas autoridades sanitarias en diferentes países ${ }^{1,4,6-10}$

A pesar de estar catalogadas por la Autoridad Europea de Seguridad Alimentaria (EFSA) como bebidas refrescantes ${ }^{7}$, la extensión de un patrón de consumo asociado al alcohol, ha provocado casos de reacciones adversas (insomnio, ansiedad o deshidratación) que han motivado a algunas organizaciones de salud a recomendar que se evite el consumo simultáneo al alcohol, durante el embarazo o la lactancia, durante actividades físicas intensas y en personas con patologías cardíacas o trastornos neurológicos; también se recomienda que no sean consumidas por niños, adolescentes o personas sensibles a la cafeína ${ }^{10-12}$. 
En el presente estudio se describe una intervención educativa, que formó parte de un programa de educación para la salud implementado por profesionales de la entidad sin ánimo de lucro Mutua Manresana, en colaboración con el comité organizador de las $\mathrm{V}$ Jornadas Tecnológicas Anuales del Instituto Lacetània, ambos de la ciudad de Manresa (Barcelona). La motivación para incluir las bebidas energéticas entre los temas a tratar en el evento fueron: 1 ) los resultados de una encuesta realizadas por la Agencia Catalana de Seguridad Alimentaria en la población general y en algunos colectivos vulnerables, que identificó un consumo medio alto en aproximadamente el $68 \%$ de los adolescentes de entre 10 y 18 años, de los cuales el 53\% acostumbra a mezclarlas con alcohol ${ }^{13}$; 2) la preocupación manifestada por los profesores del centro de estudios en que se planificó la intervención, dado el consumo habitual de estos refrescos entre los estudiantes del mismo y 3 ) las recomendaciones de algunas agencias de seguridad alimentaria de mejorar la educación de niños, adolescentes y padres en los riesgos para la salud que puede suponer el abuso de estas sustancias ${ }^{14,15}$.

La Educación para la Salud (EpS) es un proceso de trasmisión de conocimientos, un proceso de aprendizaje significativo y un medio para estimular cambios en las conductas y en el estilo de vida ${ }^{16,17}$ de las personas. En esta investigación, el proceso de aprendizaje se basa en la comparación y valoración de los conocimientos adquiridos con los conocimientos previos a las intervenciones educativas, aprovechando rasgos cognitivos individuales de los participantes, para incidir positivamente en la adquisición de conocimientos relacionados con la salud. Algunos de estos rasgos son las estrategias cognitivas individuales que se combinan entre sí, para configurar el estilo de aprendizaje preferente de un individuo, aceptado como su forma de aprender y de dar significado a la información recibida ${ }^{18-20}$.
La importancia de la EpS como una vía educativa y la influencia de los estilos de aprendizaje en la adquisición de los conocimientos, sirvieron de base para plantear la presente investigación, que tuvo como objetivos: diseñar un taller educativo sobre las características de las bebidas energéticas y las consecuencias de su consumo para la salud, utilizando técnicas de enseñanza afines a las estrategias de aprendizaje preferente de un grupo de adolescentes; así como evaluar el impacto del taller en el nivel de conocimientos de los participantes acerca de las bebidas energéticas.

\section{MATERIAL Y MÉTODOS}

Características del estudio y muestra: De septiembre de 2014 a abril de 2015 se realizó un estudio de intervención con evaluación pre- y post- intervención. A través de un muestreo intencional, se convocó a los 103 estudiantes matriculados en cuarto curso de enseñanza secundaria del Instituto Lacetània de Manresa en Barcelona, que aceptaron participar voluntariamente. Estos respondieron el cuestionario VARK (Visual/Auditivo/Lecto-escritor/Kinestésico) ${ }^{19,20}$ de estilo de aprendizaje. El instrumento consta de 16 preguntas de opción múltiple con cuatro posibles respuestas; el encuestado tiene la opción de no contestar a las preguntas cuyas respuestas no se apliquen a sus preferencias individuales. A través del análisis de las respuestas se diagnosticaron los estilos de aprendizaje: visual, auditivo, lecto-escritor y quinestésico, cuyas características principales se resumen en la Tabla 1. El cuestionario además permite identificar combinaciones de estos que constituyen los estilos bimodal, trimodal o multimodal.

Tabla 1. Principales características que distinguen los rasgos del aprendizaje de los individuos con los estilos de aprendizaje identificados a través del cuestionario VARK.

\section{Estilos de aprendizaje}

Visual

Auditivo

Lecto-escritor

Quinestésico
Rasgos del aprendizaje

Preferencia por las maneras gráficas y simbólicas de representar la información: diagramas, gráficas, colores, cuadros, textos escritos con diferentes tipos de letra y diferentes arreglos espaciales.

Preferencia por escuchar la información: debates, argumentos, discusiones, conversaciones, audios, seminarios y música.

Preferencia por la información impresa en forma de palabras: libros, textos, lecturas, retroalimentaciones escritas, toma de notas, ensayos y bibliografías.

Preferencia perceptual relacionada con el uso de la experiencia y la práctica, ya sea real o simulada: ejemplos de la vida real, cátedras de invitados, demostraciones, actividad física, construcciones, juego de roles y modelos de trabajo. 
Los 103 estudiantes respondieron un cuestionario autoadministrado, elaborado por consenso entre expertos profesionales de la salud y la educación. La encuesta diseñada para evaluar el nivel de conocimientos de los participantes acerca de las características de las bebidas energéticas, consta de cinco preguntas de selección múltiple y una de respuesta abierta, relacionada con: la definición de las bebidas energéticas, los posibles efectos de su consumo para la salud, los grupos poblacionales que deben evitar el consumo y el contenido en cafeína de estas y otras bebidas o alimentos (ver fichero adicional).

Los resultados obtenidos en la aplicación de los cuestionarios, sirvieron de base para diseñar un taller educativo sobre las bebidas energéticas, en el que participaron los estudiantes del centro que aspiraban a cursar el bachillerato en ciencias. Los estilos de aprendizaje identificados permitieron conocer las estrategias de aprendizaje preferentes de la mayoría de los estudiantes y seleccionar las técnicas de enseñanza acordes a estas estrategias y por tanto recomendadas para facilitar la adquisición de conocimientos en los individuos con estos estilos ${ }^{19,20}$; y la encuesta sobre las bebidas energéticas estableció el nivel de los conocimientos previos a la intervención educativa. Estas técnicas fueron utilizadas en el diseño del taller (Tabla 2).
Características de la intervención educativa: Consistió en la realización del taller "Bebidas Energéticas, precio y peaje de esta energía". Organizado en tres etapas:

1. Etapa de diseño y planificación: Se definieron los objetivos, se informó a los estudiantes y profesores del centro educativo de las características de la intervención, se obtuvo la autorización del centro y el consentimiento informado de los participantes. La etapa concluyó con la aplicación del cuestionario VARK de estilo de aprendizaje y del cuestionario de conocimientos acerca de las bebidas energéticas (aplicación preintervención).

2. Etapa de intervención: Se realizó el taller de dos horas de duración, que incluyó la presentación de los objetivos y del contenido, seguida de la ejecución de las técnicas de enseñanza que se relacionan en la Tabla 2. Finalmente se realizó un resumen de los contenidos impartidos.

3. Etapa de evaluación del taller. Con la intención de valorar el grado de satisfacción de los participantes respecto a la actividad educativa, durante la media hora posterior al taller, se les convidó a responder bien, regular o mal a las siguientes preguntas: "¿Cómo evalúas la habilidad del ponente para transmitir la información?", "¿Cómo evalúas la cantidad y calidad del contenido recibido?" y

Tabla 2. Técnicas de enseñanza y actividad realizada en el taller según las estrategias de aprendizaje preferentes para los estilos de aprendizaje predominantemente identificados.

\begin{tabular}{|c|c|c|}
\hline Estilos de aprendizaje & Estrategias de aprendizaje preferentes & Técnica de enseñanza utilizada en el taller \\
\hline \multirow[t]{2}{*}{ Auditivo } & $\begin{array}{l}\text { Lectura de textos en alta voz y discusión } \\
\text { del contenido de lo leído. }\end{array}$ & $\begin{array}{l}\text { Lectura guiada. Lee la etiqueta de algunas de las } \\
\text { bebidas energéticas y responde a la siguiente } \\
\text { pregunta: ¿Quién y cómo nos convidan a } \\
\text { ingerirlas? }\end{array}$ \\
\hline & Debates y discusiones. & $\begin{array}{l}\text { Lluvia de ideas. A partir de la proyección de } \\
\text { imágenes que ejemplifican las situaciones de } \\
\text { consumo habitual de las bebidas energéticas. }\end{array}$ \\
\hline \multirow{2}{*}{ Quinestésico } & $\begin{array}{l}\text { Manipulación manual de objetos. } \\
\text { Necesidad de desplazarse, experimentar } \\
\text { y crear. }\end{array}$ & $\begin{array}{l}\text { Puzle. Construye una frase que responda a } \\
\text { la siguiente pregunta: ¿Qué nos aportan las } \\
\text { bebidas energéticas? }\end{array}$ \\
\hline & $\begin{array}{l}\text { Desarrollar ejemplos. } \\
\text { Participar en situaciones que involucren } \\
\text { la experiencia y la práctica simulada o } \\
\text { real. }\end{array}$ & $\begin{array}{l}\text { Juego de roles. A partir de un listado de } \\
\text { alimentos y bebidas, que un adolescente } \\
\text { consume en un día: Calcula cuántos Red Bull } \\
\text { podrías beber sin superar la dosis de cafeína } \\
\text { diaria recomendada. }\end{array}$ \\
\hline
\end{tabular}


"¿Cómo evalúas la efectividad del taller para responder a tus expectativas?". La etapa de evaluación concluyó entre 10 y 15 días posteriores a la intervención, en que se realizó una segunda aplicación del cuestionario de conocimientos sobre las bebidas energéticas (aplicación posintervención). El análisis de los resultados permitiría valorar si la intervención mejoró en nivel de conocimientos demostrados previamente.

El tiempo de aplicación de la encuesta sobre las bebidas energéticas varió de 30 minutos en la primera aplicación a 21,5 minutos en la segunda. Los valores de consistencia interna global para ambas aplicaciones, refuerzan la fiabilidad de los resultados (cuestionario preintervención: $\alpha$-Cronbach $=0,724 ;$ y cuestionario posintervención: $a$-Cronbach $=0,806)$. La evolución del nivel de conocimientos se evaluó comparando el número de respuestas correctas en las dos aplicaciones del cuestionario.

Aspectos éticos: La aplicación de los cuestionarios fue autorizada por el equipo docente y el comité organizador de las V Jornadas Tecnológicas Anuales del Instituto Lacetània, en el marco de las cuales se realizó el taller. Los encuestadores trasmitieron a los estudiantes orientaciones precisas para responder los cuestionarios, les informaron que sus respuestas serían utilizadas en una investigación científica, garantizaron el anonimato de los datos y que se aplicara el sistema de codificación que permitiría comparar los resultados de las dos aplicaciones.

Se añadió a los cuestionarios un encabezamiento para indicar la edad, sexo, peso y altura. El comité organizador de las jornadas, contaba con la autorización a través de consentimiento informado de los estudiantes, o de sus padres en caso de los menores de edad, para contestar las encuestas y participar del taller. La intervención es parte del trabajo de tesis doctoral de uno de los autores, aprobado por el Comité Científico de Evaluación del Programa de Doctorado en Salud y Calidad de Vida de la Universidad de Vic - Universidad Central de Cataluña.

Análisis estadístico: Las respuestas fueron analizadas con el programa SPSS Versión 15.0 para Windows. Todos Ios intervalos de confianza fueron calculados al 95\%.

Se calcularon las frecuencias absolutas y relativas, así como las medias y Desviación Estándar (DE) necesarias para describir las características demográficas de la muestra y evaluar los conocimientos en las dos aplicaciones de la encuesta sobre las bebidas energéticas. La evolución en el nivel de conocimientos se obtuvo a partir de la variación entre el número de respuestas correctas entre el pre-post intervención para el total de la muestra y diferenciando por tipo de estilo de aprendizaje. Para explorar la relación entre los estilos de aprendizaje con el nivel de conocimientos adquiridos tras la intervención, se relacionaron estas variables a través de tablas de contingencia.

En el análisis del cuestionario VARK se consideró que un estilo era dominante cuando fue elegido en más del $50 \%$ de las respuestas y si dos o más estilos alcanzan este porcentaje, la categoría correspondería a un estilo bimodal, trimodal o multimodal (combinación de dos, tres o más preferencias dominantes respectivamente). En caso de que ningún estilo obtuviera el $50 \%$, el umbral de aceptación se bajó al $40 \%$ (preferencia fuerte).

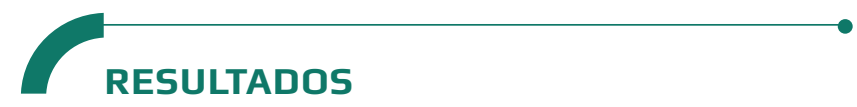

De los 103 estudiantes de cuarto curso de secundaria básica obligatoria encuestados en la etapa de diseño y planificación de la intervención educativa, 93 respondieron correctamente los cuestionarios. Los participantes eran en un $58,1 \%$ del sexo masculino y tenían una edad media de $15,4(D E=0,7)$.

En este grupo de estudiantes se identificaron los cuatro estilos de aprendizaje descritos en el modelo VARK, siendo los estilos auditivo, quinestésico y combinaciones bimodales de estos dos estilos, los predominantes en el colectivo. De ahí que para el diseño del taller se seleccionaran técnicas afines a individuos con preferencias por escuchar información y por participar en actividades prácticas (Tabla 1).

En la Tabla 3 se muestra la distribución de los estudiantes, según las respuestas al cuestionario preintervención. Un elevado número de estudiantes consideró las bebidas energéticas como isotónicas para deportistas (76,3\%) e indicadas para recuperar energía después de realizar una actividad física (67,7\%). Entre los posibles efectos negativos atribuibles a estos productos, más de la mitad de los encuestados $(52,7 \%)$ indicó que pueden provocar adicción, aunque mayoritariamente $(74,2 \%)$ admiten que pueden ser consumidas esporádicamente y en pequeñas cantidades. La mayoría de estudiantes encuestados (94,6\%-97,8\%) reconoció que el café exprés, la Coca Cola y las bebidas energéticas, pueden ser fuentes de cafeína, sin embargo, un número considerable $(49,5 \%)$ afirma que el té o el chocolate blanco no la contienen. Al preguntar acerca de las posibles reacciones o consecuencias del consumo de bebidas energéticas para la salud, la mayoría (76,3\%) afirma que pueden provocar nerviosismo, una proporción considerable cree que pueden provocar insomnio $(48,4 \%)$, pero también son muchos los que consideran que aportan energía para el deporte (61,3\%) y que son un estímulo para el ocio puesto que "provocan deseos de ir de fiesta" $(46,2 \%)$. 
Tabla 3. Distribución absoluta (n) y porcentual de los estudiantes según sus respuestas al cuestionario preintervención.

\begin{tabular}{|c|c|c|}
\hline \multicolumn{2}{|r|}{ Opción de respuesta seleccionada } & $\mathrm{n}(\%)$ \\
\hline Pregunta 1 & $\begin{array}{l}\text { Definición: "bebidas sin alcohol con propiedades estimulantes" } \\
\text { Son bebidas isotónicas para deportistas } \\
\text { Nos permiten recuperar energía después de realizar una actividad física }\end{array}$ & $\begin{array}{l}64(68,8 \%) \\
71(76,3 \%) \\
63(67,7 \%)\end{array}$ \\
\hline Pregunta 2 & $\begin{array}{l}\text { Se pueden consumir esporádicamente y en pequeñas cantidades } \\
\text { Pueden provocar adicción }\end{array}$ & $\begin{array}{l}69(74,2 \%) \\
49(52,7 \%)\end{array}$ \\
\hline Pregunta 3 & $\begin{array}{l}\text { Estudiantes que reconocen haber escuchado el término cafeinismo, que definen } \\
\text { como: } \\
\text { - Una adicción a la cafeína } \\
\text { - Una sustancia que activa, perjudica o aporta energía a nuestro cuerpo } \\
\text { - Un proceso que nos estimula, nos mantiene despiertos o nos aporta energía }\end{array}$ & $\begin{array}{c}52(55,9 \%) \\
16(17,2 \%) \\
9(9,7 \%) \\
5(5,4 \%)\end{array}$ \\
\hline Pregunta 4 & $\begin{array}{l}\text { Reconocen que el café exprés, la Coca Cola y las bebidas energéticas contienen } \\
\text { cafeína } \\
\text { Consideran que el té y el chocolate blanco no contienen cafeína } \\
\text { Consideran que el café descafeinado, el té y el chocolate blanco son las sustancias } \\
\text { con menor cantidad de cafeína } \\
\text { Consideran que el chocolate negro, el café exprés, las bebidas energéticas y la } \\
\text { Coca Cola son las sustancias con mayor cantidad de cafeína }\end{array}$ & $\begin{array}{l}88-91(94,6-97,8 \%) \\
\begin{array}{l}46(49,5 \%) \\
24(25,8 \%) \\
33(35,5 \%)\end{array}\end{array}$ \\
\hline Pregunta 5 & $\begin{array}{l}\text { Entre las reacciones o consecuencias más frecuentes del consumo de bebidas } \\
\text { energéticas destacan: } \\
\text { - Nerviosismo } \\
\text { - Aportan energía para realizar deporte } \\
\text { - Provocan insomnio } \\
\text { - Nos provocan deseos de ir de fiesta }\end{array}$ & $\begin{array}{l}71(76,3 \%) \\
57(61,3 \%) \\
45(48,4 \%) \\
43(46,2 \%)\end{array}$ \\
\hline Pregunta 6 & $\begin{array}{l}\text { En los grupos en que no se recomienda el consumo destacan: } \\
\text { - Embarazadas } \\
\text { - Personas con enfermedades cardiacas o sensibles a la cafeína } \\
\text { - Niños } \\
\text { - Adolescentes }\end{array}$ & $\begin{array}{l}71(76,3 \%) \\
70(75,3 \%) \\
65(69,9 \%) \\
26(28,0 \%)\end{array}$ \\
\hline
\end{tabular}

En el taller educativo participaron 52 estudiantes de los 103 que participaron en la encuesta previa con una distribución similar en edad y sexo a la muestra inicial $(61,6 \%$ eran chicos y tenían una edad media 15,4 años). Entre estos, los estilos de aprendizaje se comportaron de manera similar al total de estudiantes, manifestando preferencias por los estilos auditivo, quinestésico y combinaciones bimodales de estos, en 41 estudiantes (78,8\%); mientras que los estilos visual, lecto-escritor y multimodal se manifestaron como preferentes en el $21,2 \%$ de los encuestados.
Según el comportamiento de los estilos de aprendizaje se identificó la lectura de textos y la participación en debates y discusiones, como estrategias de aprendizaje preferentes para los individuos con estilo auditivo; seleccionando la lectura guiada y la lluvia de ideas como técnicas de enseñanza afines. Mientras que los individuos con estilo de aprendizaje quinestésico mostraron preferencias por la manipulación de objetos y actividades relacionadas con la práctica real o simulada; en este caso se seleccionaron el puzle y los juegos de rol (Tabla 2). Todos los estudiantes con independencia de su estilo de aprendizaje participaron en las cuatro técnicas de enseñanza. 
En la Tabla 4 se muestran los cambios en el número de respuestas correctas entre el pre- y el post- taller tanto para el total de individuos intervenidos como distinguiendo por estilo de aprendizaje. En general, se aumentó el número de respuestas correctas entre ambos momentos para el total de la muestra, observándose un mayor incremento para las preguntas que abordaban la definición de bebida energética (Pregunta 1 [Tabla 3]) y la definición del cafeinismo (Pregunta 3 [Tabla 3]). Al distinguir por estilo de aprendizaje, se observó un aumento en el número de preguntas correctas entre la pre- y la post- intervención, siendo significativamente $(p<0,05)$ mayor para los de estilo de aprendizaje visual, lecto escrito y multimodal con excepción para la pregunta que abordaba la definición de bebida energética que fue mayor en los estudiantes con estilo de aprendizaje preferentemente auditivo, quinestésico y bimodal.

El taller fue evaluado por todos los participantes: el 100\% expresó que la actividad había cumplido sus expectativas y el 98 y 93,3\% respectivamente, evaluaron de satisfactoriamente la intervención del ponente y la calidad del contenido impartido.

\section{DISCUSIÓN}

La necesidad de educar a los jóvenes y adolescentes respecto a las propiedades de las bebidas energéticas y a los posibles efectos de su consumo para la salud, es un reclamo actual de algunas instituciones y profesionales sanitarios ${ }^{1,10}$. Se puso de manifiesto en las respuestas al cuestionario preintervención, que mostró el desconocimiento de los estudiantes acerca de algunas de las características y propiedades de estas sustancias, así como de sus posibles efectos negativos para la salud. Mientras que los resultados de la encuesta posintervención apuntan a una elevación en nivel de conocimientos, reforzando la validez de la intervención realizada como una opción de EpS en este colectivo.

La carencia de estudios fiables que profundicen en las propiedades de las bebidas energéticas y de otras sustancias que contienen cafeína añadida, unido a opiniones contradictoras al respecto, apuntan a la necesidad de profundizar en su estudio ${ }^{4,9,21}$. Ya en 1983, el Comité Científico de la Alimentación Humana (SCF, por sus siglas en inglés) evaluó los posibles efectos de la cafeína, componente principal de las bebidas energéticas, concluyendo que posee ciertas propiedades farmacológicas y biológicas en animales y en humanos, pero que no se conocen sus mecanismos estimulantes en el sistema nervioso central ${ }^{22}$. En 2011, la EFSA publicó dos opiniones científicas relacionadas con la cafeína, en la que se valoran sus efectos sobre diferentes procesos fisiológicos, señalando una relación causal con cambios en la atención y estado de alerta, pero no con las modificaciones del metabolismo lipídico o reducción de la grasa corporal $^{23,24}$. La relación ente el consumo de bebidas energéticas y los procesos de atención se ha encontrado en otros estudios, que no reconocen que estos productos faciliten ni mejoren el desempeño cognitivo ${ }^{5}$.

Tabla 4. Distribución de los estudiantes según el número y \% de respuestas correctas en las dos aplicaciones del cuestionario: preintervención y posintervención.

\begin{tabular}{|c|c|c|c|c|c|c|}
\hline \multirow[t]{3}{*}{ Preguntas sobre las bebidas energéticas } & \multicolumn{6}{|c|}{ Distribución de los estudiantes según el número y $\%$ de respuestas correctas } \\
\hline & \multicolumn{2}{|c|}{$\begin{array}{l}\text { Total } \\
(n=52)\end{array}$} & \multicolumn{2}{|c|}{$\begin{array}{l}\text { Estudiantes con } \\
\text { estilo de aprendizaje } \\
\text { preferentemente auditivo, } \\
\text { quinestésico y bimodal } \\
(\mathrm{n}=41)\end{array}$} & \multicolumn{2}{|c|}{$\begin{array}{l}\text { Estudiantes con } \\
\text { estilo de aprendizaje } \\
\text { preferentemente visual, } \\
\text { lecto-escritor y multimodal } \\
\qquad(\mathrm{n}=11)\end{array}$} \\
\hline & Preintervención & Posintervención & Preintervención & Posintervención & Preintervención & Posintervención \\
\hline Definición & $25(48,1 \%)$ & $42(80,7 \%)$ & $23(44,2 \%)$ & $37(71,1 \%)$ & $2(18,2 \%)$ & $5(45,4 \%)$ \\
\hline Posibles efectos sobre la salud & $29(55,8 \%)$ & $35(67,3 \%)$ & $25(48,1 \%)$ & $28(53,8 \%)$ & $4(36,4 \%)$ & $7(63,6 \%)$ \\
\hline Concepto de cafeinismo & $28(52,8 \%)$ & 52 (100\%) & $23(44,2 \%)$ & $41(78,8 \%)$ & $5(45,4 \%)$ & $11(100 \%)$ \\
\hline Contenido de cafeína en bebidas y alimentos & $19(36,5 \%)$ & $30(57,7 \%)$ & $16(30,8 \%)$ & $24(46,1 \%)$ & $3(27,3 \%)$ & $6(54,5 \%)$ \\
\hline Efectos negativos del consumo para la salud & $29(55,8 \%)$ & $40(76,9 \%)$ & $23(44,2 \%)$ & $32(61,5 \%)$ & $6(54,5 \%)$ & $8(72,7 \%)$ \\
\hline Recomendaciones para el consumo & $32(61,5 \%)$ & $45(86,5 \%)$ & $27(51,9 \%)$ & $37(71,1 \%)$ & $5(45,4 \%)$ & $8(72,7 \%)$ \\
\hline
\end{tabular}


Los rasgos que definen a las bebidas energizantes identificados por la mayor parte de los encuestados, están en línea con la creencia popular y con las campañas publicitarias, que presentan estos refrescos como sustancias estimulantes, que aportan energía y que prolongan el estado de alerta. Estudios realizados en diferentes países ${ }^{1,2,3}$ y encuestas aplicadas en los últimos años, señalan a los jóvenes y adolescentes como los grupos de edad más propensos al consumo de la mayoría de las bebidas energizantes ${ }^{13}$. Estos datos se corresponden con el escaso número de encuestados que en este estudio incluyó a los adolescentes entre los grupos en que se recomienda un consumo moderado y que indican mayoritariamente que las bebidas energéticas pueden ser consumidas esporádicamente y en pequeñas cantidades (Tabla 3).

Entre las reacciones o consecuencias de la ingesta de bebidas energéticas mayoritariamente señaladas por los participantes, destacan que pueden provocar nerviosismo o crear adicción. A pesar de que los datos actuales disponibles no permiten definir con garantías una asociación causal entre el consumo de bebidas energéticas y los efectos sobre la salud que se le atribuyen, algunas investigaciones sugieren que pueden provocar reacciones adversas sobre todo asociadas al alcohol, que actúan como estimulantes sobre el estado de vigilia y la capacidad de reacción o que deben ser catalogadas como sustancias estimulantes o drogas de abuso y no como bebidas refrescantes ${ }^{1,7,9,21-23}$

El presente estudio fue diseñado con la intención de evaluar e influir en el nivel de conocimientos de los adolescentes acerca de las bebidas energéticas, y no para describir los patrones de consumo, evitando predisponerlos negativamente hacia la intervención educativa. Como líneas futuras, se plantea evaluar el consumo de bebidas energizantes en todos los estudiantes del centro y valorar la efectividad de las intervenciones educativas para mejorar el nivel de conocimientos respecto a las propiedades de estos refrescos, así como evaluar si estas actividades pueden ocasionar cambios de conducta, si fuera necesario.

Los resultados expresados en la Tabla 4 indican que en el cuestionario posintervención, aumentó el número de respuestas correctas en todas las preguntas. De estos valores se puede inferir que el empleo de técnicas de enseñanza reconocidas como preferentes para los individuos con estilo de aprendizaje auditivo y quinestésico ${ }^{19,20}$, también facilitó que los estudiantes con estilo de aprendizaje visual y lectoescritor, mejoraran en sus conocimientos, sugiriendo que el papel determinante de los estilos de aprendizaje en la adquisición de conocimientos podría estar reforzado por otros aspectos del aprendizaje $\mathrm{e}^{18,25-28}$, como son el grado de satisfacción y cumplimiento de las expectativas de los adolescentes respecto a la actividad educativa expresadas al evaluarla. Otro aspecto que podría haber influido en los resultados posintervención, es el grado de motivación de los participantes por discutir un tema de actualidad sobre el que (según las opiniones expresadas) reciben a través de la publicidad en los medios de comunicación, mensajes que contradicen las recomendaciones de los profesionales de la salud.

La dinámica del taller a través de actividades participativas (lectura guiada, Iluvia de ideas, puzle y juego de roles) que favorecen el debate y la discusión, además de generar un ambiente de aprendizaje en que los adolescentes se sintieron a gusto, sugiere que los conocimientos expresados a través de la encuesta y los criterios expuestos en las discusiones podrían estar relacionados con la experiencia en el consumo de bebidas energéticas.

Uno de los temas debatidos en el taller es la honestidad de la publicidad asociada al contenido de las etiquetas. A través de algunas de sus opiniones, los estudiantes criticaron que actúan como una invitación y un reclamo para el consumo. Estas opiniones son reconocidas por publicaciones científicas que critican que, en los envases de bebidas energéticas, no conste la composición cualitativa ni cuantitativa del producto, contraviniendo las recomendaciones de las agencias de seguridad alimentaria, o que se debe revisar el modo en que están catalogadas dado su poder estimulante y su probable capacidad de crear adicción $n^{7,9}$.

Los resultados analizados refuerzan el papel de la EpS en la adquisición de conocimientos que podrían influir en las conductas y en el estilo de vida de los adolescentes. La relación estadísticamente significativa entre el cambio en el nivel de los conocimientos y los estilos de aprendizaje de los participantes en el taller, refuerzan que el modelo utilizado (adaptado a las preferencias de aprendizaje identificadas), resultó una vía eficaz para garantizar la calidad de la EpS y de estimular la participación e interés de los participantes. El empleo de técnicas participativas como instrumento de formación permite generar un proceso de discusión y reflexión, que enriquecen el conocimiento individual potenciando el conocimiento del colectivo, para desarrollar una experiencia de reflexión educativa común.

En relación a las bebidas energéticas como objeto de estudio, la intervención refuerza la necesidad de estudiar la relación entre la ingesta de cafeína y sus posibles efectos sobre la salud y ratifica la importancia de realizar acciones educativas capaces de corregir las percepciones erróneas que poseen los adolescentes, a la vez que se refuerce la importancia de una alimentación e hidratación adecuadas. 


\section{CONCLUSIONES}

Un gran número de adolescentes tiene poco o nulo conocimiento sobre las características de bebidas energéticas y las consecuencias de su consumo para la salud. Tras un taller, a través de técnicas de enseñanza participativas y cooperativas afines a las estrategias de aprendizaje preferentes de los participantes, aumenta considerablemente el nivel de conocimientos de los asistentes.

\section{FICHEROS ADICIONALES}

Encuesta de conocimientos sobre las Bebidas Energéticas y su implicación en la Salud.

http://www.renhyd.org/index.php/renhyd/article/ downloadSuppFile/228/1847

\section{$\longrightarrow$ \\ CONFLICTO DE INTERESES}

Las autoras expresan que no hay conflictos de interés al redactar el manuscrito.

\section{REFERENCIAS}

(1) Ramón-Salvador DM, Cámara-Flores JM, Cabral-León FJ, Juárez-Rojop IE, Díaz-Zagoya JC. Consumo de bebidas energéticas en una población de estudiantes universitarios del estado de Tabasco, México. Salud Tab. 2013; 19(1): 10-4.

(2) Petit A, Karila L, Lejoyeux M. L'abus de boissons énergisantes présente-t-il un risque? Presse Med. 2015; 44(3): 261-70.

(3) Babu KM, Church RJ, Lewander W. Energy Drinks: The New EyeOpener For Adolescents. Clin Pediatr Emerg Med. 2008; 9(1): 35-42.

(4) Castellanos RA, Rossana MR, Frazer GG. Efectos fisiológicos de las bebidas energizantes. Rev Fac Cienc Méd. 2006; 3(1): 43-9.

(5) Mejía OMA, Galvis-Pedrosa CF, Heredia-Mazuera HA, RestrepoPinzón A. Efecto de las bebidas energizantes con base en taurina y cafeína sobre la atención sostenida y selectiva en un grupo de 52 adultos jóvenes entre 18 y 22 años de la ciudad de Bogotá. Revista Iberoamericana de Psicología: Ciencia y Tecnología. 2008; 1(1): 73-85.
(6) Persad LAB. Energy drinks and the neurophysiological impact of caffeine. Front Neurosci. 2011; 5(116): 1-8.

(7) Pérez de Ciriza JA, Pinillos MA, Aldaz J. Drogas emergentes y alimentos. An Sist Sanit Navar. 2013; 36(2): 329-32.

(8) Universidad Camilo José Cela. Estudio de la UCJC sobre los efectos negativos y positivos de las bebidas energéticas en los deportistas [Internet]. Universidad Camilo José Cela. 2014 [citado 16 de marzo de 2016]. Disponible en: http://www.ucjc. edu/2014/10/estudio-de-la-ucjc-sobre-los-efectos-negativosy-positivos-de-las-bebidas-energeticas-en-los-deportistas/

(9) Pardo R, Alvarez Y, Barral D, Farré M. Cafeína: un nutriente, un fármaco o una droga de abuso. Adicciones. 2007; 19(3): 225-38.

(10) L'Agència de Salut Pública de Catalunya. Recomanacions de I'ASPCAT sobre el consum de begudes energètiques [Internet]. Generalitat de Catalunya. 2014 [citado 16 de marzo de 2016]. Disponible en: http://www.gencat.cat/salut/acsa/html/ca/ dir3619/doc36018.html

(11) Nawrot P, Jordan S, Eastwood J, Rotstein J, Hugenholtz A, Feeley $M$. Effects of caffeine on human health. Food Addit Contam. 2003; 20(1): 1-30.

(12) Iglesias-Lepine ML, Epelde F, Espinosa J, Mariñosa $M$. [Consumption of energy drinks with alcohol, hazardous mixture]. Med Clin. 2013; 141(3): 135-6.

(13) Agència de Salut Pública de Catalunya. Informe aprovat pel Comitè Científic Assessor de Seguritat Alimentària: La seguretat de I'ús de la cafeïna en I'alimentació. Barcelona, España: Agència de Salut Pública de Catalunya; 2013.

(14) Committee on Nutrition and the Council on Sports Medicine and Fitness. Sports drinks and energy drinks for children and adolescents: are they appropriate? Pediatrics. 2011; 127(6): 1182-9.

(15) Seifert SM, Schaechter JL, Hershorin ER, Lipshultz SE. Health effects of energy drinks on children, adolescents, and young adults. Pediatrics. 2011; 127(3): 511-28.

(16) Fierro A, Alfaro M. Educación para la salud grupal. Form Act Pediatr Aten Prim. 2013; 6: 75-86.

(17) Granado de la Orden S, Serrano C, Belmonte S. Quality of life, dependency and mental health scales of interest to nutritional studies in the population. Nutr Hosp. 2015; 31(Suppl 3): 265-71.

(18) Muñetón MJB, Pinzón MAV, Alarcón LLA, Olaya CIB. Estilos y estrategias de aprendizaje: una revisión empírica y conceptual de los últimos diez años. Pensam Psicol. 2012; 10(1): 129-44.

(19) Fleming ND. I'm different, not dumb. Modes of presentation (VARK) in the tertiary classroom. En: Zelmer A, editor. Research and Development in Higher Education, Proceedings of the 1995 Annual Conference of the Higher Education and Research Development Society of Australasia (HERDSA). HERDSA; 1995. p. 308-13.

(20) Fleming N, Baume D. Learning Styles Again: VARKing up the right tree! Educ Dev. 2006; 7(4): 4-7. 
(21) Ballistreri MC, Corradi-Webster CM. El uso de bebidas energizantes en estudiantes de educación física. Rev Latinoam Enfermagem. 2008; 16(especial): 558-64.

(22) Commission of the European Communities. Report of the Scientific Committee for Food on caffeine. En: Reports of the Scientific Committee for Food (Fourteenth series). Bruselas, Bélgica: Commission of the European Communities; 1983. p. 41-6.

(23) EFSA Panel on Dietetic Products, Nutrition and Allergies (NDA). Scientific Opinion on the substantiation of health claims related to caffeine and increased fat oxidation leading to a reduction in body fat mass (ID 735, 1484), increased energy expenditure leading to a reduction in body weight (ID 1487), increased alert: Caffeine related health claims. EFSA Journal. 2011; 9(4): 2054.

(24) EFSA Panel on Dietetic Products, Nutrition and Allergies (NDA). Scientific Opinion on the substantiation of health claims related to caffeine and increase in physical performance during short-term high-intensity exercise (ID 737, 1486, 1489), increase in endurance performance (ID 737, 1486), increase in endurance capacity (ID 1488) and reduction in the rated perceived exertion/effort during exercise (ID 1488, 1490) pursuant to Article 13(1) of Regulation (EC) No 1924/2006. EFSA Journal. 2011; 9(4): 2053.

(25) Sánchez V, Fornons D, Aguilar A, Vaqué C, Milà R. Evolución de los conocimientos acerca de los trastornos del comportamiento alimentario, a través de una intervención educativa en estudiantes de enseñanza post-obligatoria. Nutr Clín Diet Hosp. 2015; 35(1): 32-41.

(26) Sánchez V, Aguilar A, Vaqué C, Mila R. Educación nutricional diseñada según las estrategias de aprendizaje preferentes de los receptores. Nutr Clín Diet Hosp. 2016; 36(1): 8-9.

(27) González B, Alonso C, Rangel R. El modelo VARK y el diseño de cursos en línea. Rev Mex Bachi Dist. 2012; 4(8): 96-103.

(28) González-Peiteado M. Los estilos de enseñanza y aprendizaje como soporte de la actividad docente. J Lear Style. 2013; 6(11): $51-70$. 